\title{
EXISTENCE AND GLOBAL EXPONENTIAL STABILITY OF POSITIVE ALMOST PERIODIC SOLUTIONS FOR A DELAYED NICHOLSON'S BLOWFLIES MODEL
}

\author{
YANLI XU
}

\begin{abstract}
This paper concerns with a class of delayed Nicholson's blowflies model with a nonlinear density-dependent mortality term. Under appropriate conditions, we establish some criteria to ensure that the solutions of this model converge globally exponentially to a positive almost periodic solution. Moreover, we give some examples and numerical simulations to illustrate our main results.
\end{abstract}

\section{Introduction}

Nicholson's blowflies equation was introduced by Nicholson [22] to model laboratory fly population. Its dynamics was later studied in [8] and [23], where this model was referred to as the Nicholsons blowflies equation [8]. The theory of the Nicholsons blowflies equation has made a remarkable progress in the past forty years with main results scattered in numerous research papers. In particular, there have been extensive studies on the problem of the existence of positive periodic solutions for the classical Nicholson's model and some generalizations with variable coefficients and delays. We refer the reader to $[5,12,13,18,21,20,24,32]$ and the references cited therein. Recently, as pointed out by L. Berezansky et al. [1], a new study indicates that a linear model of density-dependent mortality will be most accurate for populations at low densities, and marine ecologists are currently in the process of constructing new fishery models with nonlinear density-dependent mortality rates. Therefore, L. Berezansky et al. [1] and W. Wang [28] proposed the following Nicholson's blowflies model with a nonlinear density-dependent mortality term:

$$
x^{\prime}(t)=-\frac{a(t) x(t)}{b(t)+x(t)}+P(t) x(t-\tau(t)) e^{-x(t-\tau(t))},
$$

Received August 5, 2013; Revised November 20, 2013.

2010 Mathematics Subject Classification. 34C25, 34K13.

Key words and phrases. Nicholson's blowflies model, nonlinear density-dependent mortality, time-varying delay, positive almost periodic solution, global exponential stability.

This work was supported by the Construct Program of the Key Discipline in Hunan Province, the Science and Technology Planning project of Technology Department of Hunan Province (Grant no. 2012FJ4300). 
where the variable coefficients and delays are continuous functions. More details on biological explanation to model (1.1) can be found in [1] and [28]. Subsequently, there have been extensive results in the literature on the most important qualitative properties of the model and its analogous equations such as existence of positive solutions, persistence, permanence, oscillation and stability; some of the results can be found in $[2,4,11,14,17,29]$. On the other hand, the variation of the environment plays an important role in many biological and ecological dynamical systems. Fink [7] and He [10] pointed out that periodically varying environment and almost periodically varying environment are foundations for the theory of nature selection. Compared with periodic effects, almost periodic effects are more frequent. In [31], it also has been shown that, in the sense of category, the "amount" of almost periodic functions (not periodic) is far more than the "amount" of continuous periodic functions. Hence, the effects of almost periodic environment on evolutionary theory have been the object of intensive analysis by numerous authors and some of these results on Nicholson's blowflies model without the nonlinear densitydependent mortality term can be found in $[3,19,27,30]$. However, to the best of our knowledge, few authors have considered the problem on positive almost periodic solutions of Nicholson's blowflies model (1.1). Hence, it is worthwhile continuing to investigate the existence and stability of positive almost periodic solutions of (1.1).

Motivated by the above discussions, in this paper, we consider the following Nicholson's blowflies model with the nonlinear density-dependent mortality term:

$$
x^{\prime}(t)=-\frac{a(t) x(t)}{b(t)+x(t)}+\sum_{j=1}^{m} \beta_{j}(t) x\left(t-\tau_{j}(t)\right) e^{-\gamma_{j}(t) x\left(t-\tau_{j}(t)\right)},
$$

where $a, b, \beta_{j}, \gamma_{j}: R \rightarrow(0,+\infty)$ and $\tau_{j}: R \rightarrow[0,+\infty)$ are almost periodic functions, and $j=1,2, \ldots, m$. Obviously, (1.2) is the recruitment-delayed model with the Rickers type birth function and the harvesting strategy Type II (cyrtoid) (see [28]), and (1.1) is a special case of (1.2) with $m=1$.

For convenience, we introduce some notations. In the following part of this paper, given a bounded continuous function $g$ defined on $R$, let $g^{+}$and $g^{-}$be defined as

$$
g^{+}=\sup _{t \in R} g(t), g^{-}=\inf _{t \in R} g(t) .
$$

It will be assumed that

$$
r=\max _{1 \leq j \leq m} \tau_{j}^{+}, a^{-}>0, b^{-}>0, \beta_{j}^{-}>0, \gamma_{j}^{-} \geq 1, j=1,2, \ldots, m .
$$

Throughout this paper, let $R_{+}$denote nonnegative real number space, $C=$ $C([-r, 0], R)$ be the continuous functions space equipped with the usual supremum norm $\|\cdot\|$, and let $C_{+}=C\left([-r, 0], R_{+}\right)$. If $x(t)$ is continuous and defined 
on $\left[-r+t_{0}, \sigma\right)$ with $t_{0}, \sigma \in R$, then we define $x_{t} \in C$, where $x_{t}(\theta)=x(t+\theta)$ for all $\theta \in[-r, 0]$.

It is biologically reasonable to assume that only positive solutions of model (1.2) are meaningful and therefore admissible. Much can be learned by considering admissible initial conditions

$$
x_{t_{0}}=\varphi, \quad \varphi \in C_{+} \text {and } \varphi(0)>0 .
$$

We denote by $x_{t}\left(t_{0}, \varphi\right)\left(x\left(t ; t_{0}, \varphi\right)\right)$ an admissible solution of admissible initial value problem (1.2) and (1.5). Also, let $\left[t_{0}, \eta(\varphi)\right)$ be the maximal right-interval of the existence of $x_{t}\left(t_{0}, \varphi\right)$.

Definition 1.1 (see $[7,10]$ ). A continuous function $u: R \rightarrow R$ is said to be almost periodic on $R$ if, for any $\epsilon>0$, the set $T(u, \epsilon)=\{\delta:|u(t+\delta)-u(t)|<$ $\epsilon$ for all $t \in R\}$ is relatively dense, i.e., for any $\epsilon>0$, it is possible to find a real number $l=l(\epsilon)>0$ with the property that, for any interval with length $l(\epsilon)$, there exists a number $\delta=\delta(\epsilon)$ in this interval such that $|u(t+\delta)-u(t)|<\epsilon$ for all $t \in R$.

From the theory of almost periodic functions in $[7,10]$, it follows that for any $\epsilon>0$, it is possible to find a real number $l=l(\epsilon)>0$, for any interval with length $l(\epsilon)$, there exists a number $\delta=\delta(\epsilon)$ in this interval such that

$$
\left\{\begin{array}{l}
|a(t+\delta)-a(t)|<\epsilon,|b(t+\delta)-b(t)|<\epsilon,\left|\beta_{j}(t+\delta)-\beta_{j}(t)\right|<\epsilon \\
\left|\tau_{j}(t+\delta)-\tau_{j}(t)\right|<\epsilon,\left|\gamma_{j}(t+\delta)-\gamma_{j}(t)\right|<\epsilon
\end{array}\right.
$$

for all $t \in R$ and $j=1,2, \ldots, m$.

Since the function $\frac{1-x}{e^{x}}$ is decreasing with the range $[0,1]$, it follows easily that there exists a unique $\kappa \in(0,1)$ such that

$$
\frac{1-\kappa}{e^{\kappa}}=\frac{1}{e^{2}} \text {. }
$$

Obviously,

$$
\sup _{x \geq \kappa}\left|\frac{1-x}{e^{x}}\right|=\frac{1}{e^{2}}
$$

Moreover, since $x e^{-x}$ increases on $[0,1]$ and decreases on $[1,+\infty)$, let $\widetilde{\kappa}$ be the unique number in $(1,+\infty)$ such that

$$
\kappa e^{-\kappa}=\widetilde{\kappa} e^{-\tilde{\kappa}}
$$

\section{Preliminary results}

In this section, some lemmas will be presented, which are of importance in proving our main results in Section 3.

Lemma 2.1. Suppose that there exists a positive constant $M>\kappa$ such that

$$
\gamma_{j}(t) M \leq \widetilde{\kappa} \text { for all } t \in R, j=1,2, \ldots, m,
$$


$\sup _{t \in R}\left\{-\frac{a(t) M}{b(t)+M}+\frac{1}{e} \sum_{j=1}^{m} \frac{\beta_{j}(t)}{\gamma_{j}(t)}\right\}<0, \inf _{t \in R, s \in[0, \kappa]}\left\{-\frac{a(t)}{b(t)+s}+\sum_{j=1}^{m} \frac{\beta_{j}(t)}{\gamma_{j}(t)} e^{-s}\right\}>0$.

Then, for $\varphi \in C^{0}=\{\varphi \mid \varphi \in C, \varphi(\theta) \in(\kappa, M)$ for all $\theta \in[-r, 0]\}$,

$$
\eta(\varphi)=\infty \text { and } x_{t}\left(t_{0}, \varphi\right) \in C^{0} \text { for } t \geq t_{0}
$$

Proof. This lemma can be proven in the similar way as in Lemma 2.1 of [15]. But for convenience of reading, we give the proof as follows. Let $x(t)=$ $x\left(t ; t_{0}, \varphi\right)$, where $\varphi \in C^{0}$. We first claim:

$$
x(t)<M \text { for all } t \in\left[t_{0}, \eta(\varphi)\right) .
$$

Suppose, for the sake of contradiction, that there exists $t_{1} \in\left(t_{0}, \eta(\varphi)\right)$ such that

$$
x\left(t_{1}\right)=M, \quad x(t)<M \text { for all } t \in\left[t_{0}-r, t_{1}\right) .
$$

Calculating the derivative of $x(t)$, together with the fact that $\sup _{x \in R} x e^{-x}=\frac{1}{e}$, (1.2), (2.2) and (2.4) imply that

$$
\begin{aligned}
0 & \leq x^{\prime}\left(t_{1}\right) \\
& =-\frac{a\left(t_{1}\right) M}{b\left(t_{1}\right)+M}+\sum_{j=1}^{m} \frac{\beta_{j}\left(t_{1}\right)}{\gamma_{j}\left(t_{1}\right)} \gamma_{j}\left(t_{1}\right) x\left(t_{1}-\tau_{j}\left(t_{1}\right)\right) e^{-\gamma_{j}\left(t_{1}\right) x\left(t_{1}-\tau_{j}\left(t_{1}\right)\right)} \\
& \leq-\frac{a\left(t_{1}\right) M}{b\left(t_{1}\right)+M}+\frac{1}{e} \sum_{j=1}^{m} \frac{\beta_{j}\left(t_{1}\right)}{\gamma_{j}\left(t_{1}\right)} \\
& <0
\end{aligned}
$$

which is a contradiction and implies that (2.3) holds.

We next show that

$$
x(t)>\kappa \text { for all } t \in\left[t_{0}, \eta(\varphi)\right) .
$$

Assume, by way of contradiction, that (2.5) does not hold. Then, there exists $t_{2} \in\left(t_{0}, \eta(\varphi)\right)$ such that

$$
x\left(t_{2}\right)=\kappa \text { and } x(t)>\kappa \text { for all } t \in\left[t_{0}-r, t_{2}\right) .
$$

Then $\kappa \leq \gamma_{j}\left(t_{2}\right) x\left(t_{2}-\tau_{j}\left(t_{2}\right)\right) \leq \gamma_{j}\left(t_{2}\right) M \leq \widetilde{\kappa}$ and hence

$$
\gamma_{j}\left(t_{2}\right) x\left(t_{2}-\tau_{j}\left(t_{2}\right)\right) e^{-\gamma_{j}\left(t_{2}\right) x\left(t_{2}-\tau_{j}(t)\right)} \geq \min \left\{\kappa e^{-\kappa}, \widetilde{\kappa} e^{-\widetilde{\kappa}}\right\}=\kappa e^{-\kappa},
$$

where $j=1,2, \ldots, m$. It follows from (2.2) and (2.6) that

$$
\begin{aligned}
0 & \geq x^{\prime}\left(t_{2}\right) \\
& =-\frac{a\left(t_{2}\right) \kappa}{b\left(t_{2}\right)+\kappa}+\sum_{j=1}^{m} \frac{\beta_{j}\left(t_{2}\right)}{\gamma_{j}\left(t_{2}\right)} \gamma_{j}\left(t_{2}\right) x\left(t_{2}-\tau_{j}\left(t_{2}\right)\right) e^{-\gamma_{j}\left(t_{2}\right) x\left(t_{2}-\tau_{j}\left(t_{2}\right)\right)}
\end{aligned}
$$




$$
\begin{aligned}
& \geq-\frac{a\left(t_{2}\right) \kappa}{b\left(t_{2}\right)+\kappa}+\sum_{j=1}^{m} \frac{\beta_{j}\left(t_{2}\right)}{\gamma_{j}\left(t_{2}\right)} \kappa e^{-\kappa} \\
& \geq \kappa \inf _{t \in R, s \in[0, \kappa]}\left\{-\frac{a(t)}{b(t)+s}+\sum_{j=1}^{m} \frac{\beta_{j}(t)}{\gamma_{j}(t)} e^{-s}\right\} \\
& >0,
\end{aligned}
$$

which is a contradiction and implies that (2.5) holds. Thus, $x(t)$ is bounded on $\left[t_{0}, \eta(\varphi)\right)$. From Theorem 2.3.1 in [9], we easily obtain $\eta(\varphi)=+\infty$. This ends the proof of Lemma 2.1 .

Lemma 2.2. Suppose (2.1) and (2.2) hold, and

$$
\sup _{t \in R}\left\{-\frac{a(t) b(t)}{(b(t)+M)^{2}}+\sum_{j=1}^{m} \beta_{j}(t) \frac{1}{e^{2}}\right\}<0 .
$$

Moreover, assume that $x(t)=x\left(t ; t_{0}, \varphi\right)$ is a solution of equation (1.2) with initial condition $\varphi \in C^{0}$ and $\varphi^{\prime}$ is bounded continuous on $[-r, 0]$. Then for any $\epsilon>0$, there exists $l=l(\epsilon)>0$, such that every interval $[\alpha, \alpha+l]$ contains at least one number $\delta$ for which there exists $N>0$ satisfying

$$
|x(t+\delta)-x(t)| \leq \epsilon \text { for all } t>N .
$$

Proof. Define a continuous function $\Gamma(u)$ by setting

$$
\Gamma(u)=\sup _{t \in R}\left\{-\left[\frac{a(t) b(t)}{(b(t)+M)^{2}}-u\right]+\sum_{j=1}^{m} \beta_{j}(t) \frac{1}{e^{2}} e^{u r}\right\}, u \in[0,1] .
$$

Then, we have

$$
\Gamma(0)=\sup _{t \in R}\left\{-\frac{a(t) b(t)}{(b(t)+M)^{2}}+\sum_{j=1}^{m} \beta_{j}(t) \frac{1}{e^{2}}\right\}<0,
$$

which implies that there exist two constants $\eta>0$ and $\lambda \in(0,1]$ such that

$$
\Gamma(\lambda)=\sup _{t \in R}\left\{-\left[\frac{a(t) b(t)}{(b(t)+M)^{2}}-\lambda\right]+\sum_{j=1}^{m} \beta_{j}(t) \frac{1}{e^{2}} e^{\lambda r}\right\}<-\eta<0 .
$$

For $t \in\left(-\infty, t_{0}-r\right]$, we add the definition of $x(t)$ with $x(t) \equiv x\left(t_{0}-r\right)$. Set

$$
\begin{aligned}
\epsilon(\delta, t)= & -\left[\frac{a(t+\delta) x(t+\delta)}{b(t+\delta)+x(t+\delta)}-\frac{a(t) x(t+\delta)}{b(t+\delta)+x(t+\delta)}\right] \\
& -\left[\frac{a(t) x(t+\delta)}{b(t+\delta)+x(t+\delta)}-\frac{a(t) x(t+\delta)}{b(t)+x(t+\delta)}\right] \\
& +\sum_{j=1}^{m}\left[\beta_{j}(t+\delta)-\beta_{j}(t)\right] x\left(t+\delta-\tau_{j}(t+\delta)\right) e^{-\gamma_{j}(t+\delta) x\left(t+\delta-\tau_{j}(t+\delta)\right)}
\end{aligned}
$$




$$
\begin{aligned}
& +\sum_{j=1}^{m} \beta_{j}(t)\left[x\left(t+\delta-\tau_{j}(t+\delta)\right) e^{-\gamma_{j}(t+\delta) x\left(t+\delta-\tau_{j}(t+\delta)\right)}\right. \\
& \left.-x\left(t-\tau_{j}(t)+\delta\right) e^{-\gamma_{j}(t+\delta) x\left(t-\tau_{j}(t)+\delta\right)}\right] \\
& +\sum_{j=1}^{m} \beta_{j}(t)\left[x\left(t-\tau_{j}(t)+\delta\right) e^{-\gamma_{j}(t+\delta) x\left(t-\tau_{j}(t)+\delta\right)}\right. \\
& \left.-x\left(t-\tau_{j}(t)+\delta\right) e^{-\gamma_{j}(t) x\left(t-\tau_{j}(t)+\delta\right)}\right], t \in R .
\end{aligned}
$$

By Lemma 2.1, the solution $x(t)$ is bounded and

$$
\kappa<x(t)<M \text { for all } t \in R \text {. }
$$

which implies that the right-hand side of (1.2) is also bounded, and $x^{\prime}(t)$ is a bounded function on $\left[t_{0}-r,+\infty\right)$. Thus, in view of the fact that $x(t) \equiv x\left(t_{0}-r\right)$ for $t \in\left(-\infty, t_{0}-r\right]$, we obtain that $x(t)$ is uniformly continuous on $R$. From (1.6) and (2.10), for any $\epsilon>0$, there exists $l=l(\epsilon)>0$, such that every interval $[\alpha, \alpha+l], \alpha \in R$, contains $\delta$ for which

$$
|\epsilon(\delta, t)| \leq \frac{1}{2} \eta \epsilon \text { for all } t \in R .
$$

Let $N_{0} \geq \max \left\{t_{0}, t_{0}-\delta\right\}$. For $t \in R$, denote

$$
u(t)=x(t+\delta)-x(t) .
$$

Then, for all $t \geq N_{0}$, we get

$$
\begin{aligned}
\frac{d u(t)}{d t}= & -\left[\frac{a(t) x(t+\delta)}{b(t)+x(t+\delta)}-\frac{a(t) x(t)}{b(t)+x(t)}\right] \\
& +\sum_{j=1}^{m} \beta_{j}(t)\left[x\left(t-\tau_{j}(t)+\delta\right) e^{-\gamma_{j}(t) x\left(t-\tau_{j}(t)+\delta\right)}\right. \\
& \left.\quad-x\left(t-\tau_{j}(t)\right) e^{-\gamma_{j}(t) x\left(t-\tau_{j}(t)\right)}\right]+\epsilon(\delta, t) .
\end{aligned}
$$

From (2.13) and the inequalities

$$
\begin{aligned}
& -\left(\frac{a(t) A}{b(t)+A}-\frac{a(t) B}{b(t)+B}\right) \operatorname{sgn}(A-B) \\
= & -\frac{a(t) b(t)}{(b(t)+A+\theta(A-B))^{2}}|A-B| \\
\leq & -\frac{a(t) b(t)}{(b(t)+M)^{2}}|A-B| \text { where } A, B \in[\kappa, M], 0<\theta<1,
\end{aligned}
$$

and

$$
\begin{aligned}
\left|s e^{-s}-t e^{-t}\right| & =\left|\frac{1-(s+\theta(t-s))}{e^{s+\theta(t-s)}}\right||s-t| \\
& \leq \frac{1}{e^{2}}|s-t| \text { where } s, t \in[\kappa,+\infty), 0<\theta<1,
\end{aligned}
$$


we obtain

$$
\begin{aligned}
& \left.D^{-}\left(e^{\lambda s}|u(s)|\right)\right|_{s=t} \\
\leq & \lambda e^{\lambda t}|u(t)|+e^{\lambda t}\left\{-\left[\frac{a(t) x(t+\delta)}{b(t)+x(t+\delta)}-\frac{a(t) x(t)}{b(t)+x(t)}\right] \operatorname{sgn}(x(t+\delta)-x(t))\right. \\
& +\mid \sum_{j=1}^{m} \beta_{j}(t)\left[x\left(t-\tau_{j}(t)+\delta\right) e^{-\gamma_{j}(t) x\left(t-\tau_{j}(t)+\delta\right)}\right. \\
& \left.\left.\quad-x\left(t-\tau_{j}(t)\right) e^{-\gamma_{j}(t) x\left(t-\tau_{j}(t)\right)}\right]+\epsilon(\delta, t) \mid\right\} \\
=\lambda & \\
& +\left|\sum_{j=1}^{\lambda t}\right| u(t) \mid+e^{\lambda t}\left\{-\left[\frac{a(t) x(t+\delta)}{b(t)+x(t+\delta)}-\frac{a(t) x(t)}{b(t)+x(t)}\right] \operatorname{sgn}(x(t+\delta)-x(t))\right. \\
& \left.\left.\quad-\gamma_{j}(t) x\left(t-\tau_{j}(t)\right) e^{-\gamma_{j}(t) x\left(t-\tau_{j}(t)\right)}\right]+\epsilon(\delta, t) \mid\right\} \\
\leq & \lambda e^{\lambda t}|u(t)|+e^{\lambda t}\left\{-\frac{a(t) b(t)}{(b(t)+M)^{2}}|u(t)|+\sum_{j=1}^{m} \beta_{j}(t) \frac{1}{e^{2}}\left|u\left(t-\tau_{j}(t)\right)\right|+|\epsilon(\delta, t)|\right\} \\
= & -\left[\frac{a(t) b(t)}{(b(t)+M)^{2}}-\lambda\right] e^{\lambda t}|u(t)|+\sum_{j=1}^{m} \beta_{j}(t) \frac{1}{e^{2}} e^{\lambda \tau_{j}(t)} e^{\lambda\left(t-\tau_{j}(t)\right)}\left|u\left(t-\tau_{j}(t)\right)\right| \\
+ & \left.e^{\lambda t}|\epsilon(\delta, t)| \text { for all } t \geq \tau_{j}(t)+\delta\right)
\end{aligned}
$$

Let

$$
U(t)=\sup _{t_{0}-r \leq s \leq t}\left\{e^{\lambda s}|u(s)|\right\} .
$$

It is obvious that $e^{\lambda t}|u(t)| \leq U(t)$, and $U(t)$ is non-decreasing.

Now, we distinguish two cases to finish the proof.

Case one.

$$
U(t)>e^{\lambda t}|u(t)| \text { for all } t \geq N_{0}
$$

We claim that

$$
U(t) \equiv U\left(N_{0}\right) \text { is a constant for all } t \geq N_{0} .
$$

Assume, by way of contradiction, that (2.19) does not hold. Then, there exists $\widetilde{t_{1}}>N_{0}$ such that $U\left(\widetilde{t_{1}}\right)>U\left(N_{0}\right)$. Since

$$
e^{\lambda t}|u(t)| \leq U\left(N_{0}\right) \text { for all } t \leq N_{0} .
$$

There must exist $\beta \in\left(N_{0}, \widetilde{t_{1}}\right)$ such that

$$
e^{\lambda \beta}|u(\beta)|=U\left(\widetilde{t_{1}}\right) \geq U(\beta),
$$


which contradicts (2.18). This contradiction implies that (2.19) holds. It follows that there exists $\widetilde{t_{2}}>N_{0}$ such that

$$
|u(t)| \leq e^{-\lambda t} U(t)=e^{-\lambda t} U\left(N_{0}\right)<\epsilon \text { for all } t \geq \tilde{t_{2}} .
$$

Case two. There is a $t_{0}^{*} \geq N_{0}$ such that $U\left(t_{0}^{*}\right)=e^{\lambda t_{0}^{*}}\left|u\left(t_{0}^{*}\right)\right|$. Then, in view of $(2.9)$ and (2.16), we get

$$
\begin{aligned}
0 \leq & \left.D^{-}\left(e^{\lambda s}|u(s)|\right)\right|_{s=t_{0}^{*}} \\
\leq & -\left[\frac{a\left(t_{0}^{*}\right) b\left(t_{0}^{*}\right)}{\left(b\left(t_{0}^{*}\right)+M\right)^{2}}-\lambda\right] e^{\lambda t_{0}^{*}}\left|u\left(t_{0}^{*}\right)\right| \\
& +\sum_{j=1}^{m} \beta_{j}\left(t_{0}^{*}\right) \frac{1}{e^{2}} e^{\lambda \tau_{j}\left(t_{0}^{*}\right)} e^{\lambda\left(t_{0}-\tau_{j}\left(t_{0}^{*}\right)\right)}\left|u\left(t_{0}^{*}-\tau_{j}\left(t_{0}^{*}\right)\right)\right|+e^{\lambda t_{0}^{*}}\left|\epsilon\left(\delta, t_{0}^{*}\right)\right| \\
\leq & \left.\left\{\frac{a\left(t_{0}^{*}\right) b\left(t_{0}^{*}\right)}{\left(b\left(t_{0}^{*}\right)+M\right)^{2}}-\lambda\right]+\sum_{j=1}^{m} \beta_{j}\left(t_{0}^{*}\right) \frac{1}{e^{2}} e^{\lambda r}\right\} U\left(t_{0}^{*}\right)+\frac{1}{2} \eta \epsilon e^{\lambda t_{0}^{*}} \\
< & -\eta U\left(t_{0}^{*}\right)+\eta \epsilon e^{\lambda t_{0}^{*}},
\end{aligned}
$$

which yields that

$$
e^{\lambda t_{0}^{*}}\left|u\left(t_{0}^{*}\right)\right|=U\left(t_{0}^{*}\right)<\epsilon e^{\lambda t_{0}^{*}} \text { and }\left|u\left(t_{0}^{*}\right)\right|<\epsilon .
$$

For any $t>t_{0}^{*}$, with the same approach as that in deriving of $(2.22)$, we can show

$$
e^{\lambda t}|u(t)|<\epsilon e^{\lambda t} \text { and }|u(t)|<\epsilon,
$$

if $U(t)=e^{\lambda t}|u(t)|$.

On the other hand, if $U(t)>e^{\lambda t}|u(t)|$ and $t>t_{0}^{*}$, we can choose $t_{0}^{*} \leq t_{3}<t$ such that

$$
U\left(t_{3}\right)=e^{\lambda t_{3}}\left|u\left(t_{3}\right)\right| \text { and } U(s)>e^{\lambda s}|u(s)| \text { for all } s \in\left(t_{3}, t\right],
$$

which, together with (2.23), yields

$$
\left|u\left(t_{3}\right)\right|<\epsilon
$$

With a similar argument as that in the proof of case one, we can show that

$$
U(s) \equiv U\left(t_{3}\right) \text { is a constant for all } s \in\left(t_{3}, t\right],
$$

which implies that

$$
|u(t)|<e^{-\lambda t} U(t)=e^{-\lambda t} U\left(t_{3}\right)=\left|u\left(t_{3}\right)\right| e^{-\lambda\left(t-t_{3}\right)}<\epsilon .
$$

In summary, there must exist $N>\max \left\{t_{0}^{*}, N_{0}, \widetilde{t_{2}}\right\}$ such that $|u(t)| \leq \epsilon$ holds for all $t>N$. The proof of Lemma 2.2 is now complete. 


\section{Main results}

In this section, we establish sufficient conditions on the existence and global exponential stability of almost periodic solutions of (1.2).

Theorem 3.1. Under the assumptions of Lemma 2.2, equation (1.2) has at least one positive almost periodic solution.

Proof. Let $v(t)=v\left(t ; t_{0}, \varphi\right)$ be a solution of equation (1.2) with initial conditions satisfying the assumptions in Lemma 2.2. We also add the definition of $v(t)$ with $v(t) \equiv v\left(t_{0}-r\right)$ for all $t \in\left(-\infty, t_{0}-r\right]$. Set

$$
\begin{aligned}
\epsilon(k, t)= & -\left[\frac{a\left(t+t_{k}\right) v\left(t+t_{k}\right)}{b\left(t+t_{k}\right)+v\left(t+t_{k}\right)}-\frac{a(t) v\left(t+t_{k}\right)}{b\left(t+t_{k}\right)+v\left(t+t_{k}\right)}\right] \\
& -\left[\frac{a(t) v\left(t+t_{k}\right)}{b\left(t+t_{k}\right)+v\left(t+t_{k}\right)}-\frac{a(t) v\left(t+t_{k}\right)}{b(t)+v\left(t+t_{k}\right)}\right] \\
& +\sum_{j=1}^{m}\left[\beta_{j}\left(t+t_{k}\right)-\beta_{j}(t)\right] v\left(t+t_{k}-\tau_{j}\left(t+t_{k}\right)\right) e^{-\gamma_{j}\left(t+t_{k}\right) v\left(t+t_{k}-\tau_{j}\left(t+t_{k}\right)\right)} \\
& +\sum_{j=1}^{m} \beta_{j}(t)\left[v\left(t+t_{k}-\tau_{j}\left(t+t_{k}\right)\right) e^{-\gamma_{j}\left(t+t_{k}\right) v\left(t+t_{k}-\tau_{j}\left(t+t_{k}\right)\right)}\right. \\
& \left.-v\left(t-\tau_{j}(t)+t_{k}\right) e^{-\gamma_{j}\left(t+t_{k}\right) v\left(t-\tau_{j}(t)+t_{k}\right)}\right] \\
& +\sum_{j=1}^{m} \beta_{j}(t)\left[v\left(t-\tau_{j}(t)+t_{k}\right) e^{-\gamma_{j}\left(t+t_{k}\right) v\left(t-\tau_{j}(t)+t_{k}\right)}\right. \\
& \left.-v\left(t-\tau_{j}(t)+t_{k}\right) e^{-\gamma_{j}(t) v\left(t-\tau_{j}(t)+t_{k}\right)}\right], t \in R,
\end{aligned}
$$

where $\left\{t_{k}\right\}$ is any sequence of real numbers. By Lemma 2.1, the solution $v(t)$ is bounded and

$$
\kappa<v(t)<M \text { for all } t \in R
$$

which implies that the right-hand side of (1.2) is also bounded, and $v^{\prime}(t)$ is a bounded function on $\left[t_{0}-r,+\infty\right)$. Thus, in view of the fact that $v(t) \equiv v\left(t_{0}-r\right)$ for $t \in\left(-\infty, t_{0}-r\right]$, we obtain that $v(t)$ is uniformly continuous on $R$. Then, from the almost periodicity of $a, b, \tau_{j}, \gamma_{j}$ and $\beta_{j}$, we can select a sequence $\left\{t_{k}\right\} \rightarrow+\infty$ such that

$$
\left\{\begin{array}{l}
\left|a\left(t+t_{k}\right)-a(t)\right| \leq \frac{1}{k},\left|b\left(t+t_{k}\right)-b(t)\right| \leq \frac{1}{k},\left|\tau_{j}\left(t+t_{k}\right)-\tau_{j}(t)\right| \leq \frac{1}{k} \\
\left|\beta_{j}\left(t+t_{k}\right)-\beta_{j}(t)\right| \leq \frac{1}{k},\left|\gamma_{j}\left(t+t_{k}\right)-\gamma_{j}(t)\right| \leq \frac{1}{k}, \quad|\epsilon(k, t)| \leq \frac{1}{k}
\end{array}\right.
$$

for all $j, t$.

Since $\left\{v\left(t+t_{k}\right)\right\}_{k=1}^{+\infty}$ is uniformly bounded and equiuniformly continuous, by the Arzala-Ascoli lemma and the diagonal selection principle, we can choose a subsequence $\left\{t_{k_{j}}\right\}$ of $\left\{t_{k}\right\}$, such that $v\left(t+t_{k_{j}}\right.$ ) (for convenience, we still denote by $\left.v\left(t+t_{k}\right)\right)$ uniformly converges to a continuous function $x^{*}(t)$ on any compact 
set of $R$, and

$$
\kappa \leq x^{*}(t) \leq M \text { for all } t \in R .
$$

Now, we prove that $x^{*}(t)$ is a solution of (1.2). In fact, for any $t \geq t_{0}$ and $\Delta t \in R$, from (3.3), we have

$$
\begin{aligned}
(3.5) & x^{*}(t+\Delta t)-x^{*}(t) \\
= & \lim _{k \rightarrow+\infty}\left[v\left(t+\Delta t+t_{k}\right)-v\left(t+t_{k}\right)\right] \\
= & \lim _{k \rightarrow+\infty} \int_{t}^{t+\Delta t}\left\{-\frac{a\left(\mu+t_{k}\right) v\left(\mu+t_{k}\right)}{b\left(\mu+t_{k}\right)+v\left(\mu+t_{k}\right)}\right. \\
& \left.+\sum_{j=1}^{m} \beta_{j}\left(\mu+t_{k}\right) v\left(\mu+t_{k}-\tau_{j}\left(\mu+t_{k}\right)\right) e^{-\gamma_{j}\left(\mu+t_{k}\right) v\left(\mu+t_{k}-\tau_{j}\left(\mu+t_{k}\right)\right)}\right\} d \mu \\
= & \lim _{k \rightarrow+\infty} \int_{t}^{t+\Delta t}\left\{-\frac{a(\mu) v\left(\mu+t_{k}\right)}{b(\mu)+v\left(\mu+t_{k}\right)}\right. \\
& \left.+\sum_{j=1}^{m} \beta_{j}(\mu) v\left(\mu+t_{k}-\tau_{j}(\mu)\right) e^{-\gamma_{j}(\mu) v\left(\mu+t_{k}-\tau_{j}(\mu)\right)}+\epsilon(k, \mu)\right\} d \mu \\
= & \int_{t}^{t+\Delta t}\left\{-\frac{a(\mu) x^{*}(\mu)}{b(\mu)+x^{*}(\mu)}\right. \\
& \left.+\sum_{j=1}^{m} \beta_{j}(\mu) x^{*}\left(\mu-\tau_{j}(\mu)\right) e^{-\gamma_{j}(\mu) x^{*}\left(\mu-\tau_{j}(\mu)\right)}\right\} d \mu \\
& +\lim _{k \rightarrow+\infty} \int_{t}^{t+\Delta t} \epsilon(k, \mu) d \mu \\
= & \int_{t}^{t+\Delta t}\left\{-\frac{a(\mu) x^{*}(\mu)}{b(\mu)+x^{*}(\mu)}+\sum_{j=1}^{m} \beta_{j}(\mu) x^{*}\left(\mu-\tau_{j}(\mu)\right) e^{-\gamma_{j}(\mu) x^{*}\left(\mu-\tau_{j}(\mu)\right)}\right\} d \mu,
\end{aligned}
$$

where $t+\Delta t \geq t_{0}$. Consequently, (3.5) implies that

$$
\frac{d}{d t}\left\{x^{*}(t)\right\}=-\frac{a(t) x^{*}(t)}{b(t)+x^{*}(t)}+\sum_{j=1}^{m} \beta_{j}(t) x^{*}\left(t-\tau_{j}(t)\right) e^{-\gamma_{j}(t) x^{*}\left(t-\tau_{j}(t)\right)} .
$$

Therefore, $x^{*}(t)$ is a solution of (1.2).

Secondly, we prove that $x^{*}(t)$ is an almost periodic solution of (1.2). From Lemma 2.2, for any $\varepsilon>0$, there exists $l=l(\varepsilon)>0$, such that every interval $[\alpha, \alpha+l]$ contains at least one number $\delta$ for which there exists $N>0$ satisfying

$$
|v(t+\delta)-v(t)| \leq \varepsilon \text { for all } t>N
$$

Then, for any fixed $s \in R$, we can find a sufficient large positive integer $N_{1}>N$ such that for any $k>N_{1}$,

$$
s+t_{k}>N, \quad\left|v\left(s+t_{k}+\delta\right)-v\left(s+t_{k}\right)\right| \leq \varepsilon .
$$


Let $k \rightarrow+\infty$, we obtain

$$
\left|x^{*}(s+\delta)-x^{*}(s)\right| \leq \varepsilon,
$$

which implies that $x^{*}(t)$ is an almost periodic solution of equation (1.2). The proof of Theorem 3.1 is now complete.

Theorem 3.2. Suppose that all conditions in Theorem 3.1 are satisfied. Let $x^{*}(t)$ be the positive almost periodic solution of equation (1.2) in Theorem 3.1. Then, $x^{*}(t)$ is globally exponentially stable, i.e., the solution $x\left(t ; t_{0}, \varphi\right)$ of $(1.2)$ with admissible initial conditions (1.5) converges exponentially to $x^{*}(t)$ as $t \rightarrow$ $+\infty$.

Proof. Let $x^{*}(t)$ be the positive almost periodic solution of equation (1.2) in Theorem 3.1. To prove Theorem 3.2, we should show the global exponential stability for $x^{*}(t)$. Since $\varphi \in C_{+}$, using Theorem 5.2.1 in [25, p. 81], we have $x_{t}\left(t_{0}, \varphi\right) \in C_{+}$for all $t \in\left[t_{0}, \eta(\varphi)\right)$. Let $x(t)=x\left(t ; t_{0}, \varphi\right)$. From (1.2) and the fact that $\frac{a(t) x}{b(t)+x} \leq \frac{a(t) x}{b(t)}$ for all $t \in R, x \geq 0$, we get

$$
\begin{aligned}
x^{\prime}(t) & =-\frac{a(t) x(t)}{b(t)+x(t)}+\sum_{j=1}^{m} \beta_{j}(t) x\left(t-\tau_{j}(t)\right) e^{-\gamma_{j}(t) x\left(t-\tau_{j}(t)\right)} \\
& \geq-\frac{a(t)}{b(t)} x(t)+\sum_{j=1}^{m} \beta_{j}(t) x\left(t-\tau_{j}(t)\right) e^{-\gamma_{j}(t) x\left(t-\tau_{j}(t)\right)} .
\end{aligned}
$$

In view of $x\left(t_{0}\right)=\varphi(0)>0$, integrating (3.10) from $t_{0}$ to $t$, we have

$$
\begin{aligned}
x(t) \geq & e^{-\int_{t_{0}}^{t} \frac{a(u)}{b(u)} d u} x\left(t_{0}\right) \\
& +e^{-\int_{t_{0}}^{t} \frac{a(u)}{b(u)} d u} \int_{t_{0}}^{t} e^{\int_{t_{0}}^{s} \frac{a(v)}{b(v)} d v} \sum_{j=1}^{m} \beta_{j}(s) x\left(s-\tau_{j}(s)\right) e^{-\gamma_{j}(s) x\left(s-\tau_{j}(s)\right)} d s
\end{aligned}
$$

(3.11) $>0$ for all $t \in\left[t_{0}, \eta(\varphi)\right)$.

We next show that there is $t_{\varphi} \in\left[t_{0}, \eta(\varphi)\right)$ such that

$$
\kappa<x(t)<M \text { for all } t \in\left[t_{\varphi}, \eta(\varphi)\right) \text {, and } \eta(\varphi)=+\infty \text {. }
$$

We first show that there exists $t_{4} \in\left[t_{0}, \eta(\varphi)\right)$ such that

$$
x\left(t_{4}\right)<M \text {. }
$$

Otherwise,

$$
x(t) \geq M \text { for all } t \in\left[t_{0}, \eta(\varphi)\right),
$$

which together with (2.2), implies that

$$
x^{\prime}(t)=-\frac{a(t) x(t)}{b(t)+x(t)}+\sum_{j=1}^{m} \frac{\beta_{j}(t)}{\gamma_{j}(t)} \gamma_{j}(t) x\left(t-\tau_{j}(t)\right) e^{-\gamma_{j}(t) x\left(t-\tau_{j}(t)\right)}
$$




$$
\begin{aligned}
& \leq-\frac{a(t) M}{b(t)+M}+\frac{1}{e} \sum_{j=1}^{m} \frac{\beta_{j}(t)}{\gamma_{j}(t)} \\
& <0 \text { for all } t \in\left[t_{0}, \eta(\varphi)\right) .
\end{aligned}
$$

This yields that $x(t)$ is bounded and monotone decreasing on $\left[t_{0}, \eta(\varphi)\right)$. Again from Theorem 2.3.1 in [6], we easily obtain $\eta(\varphi)=+\infty$. Then, (3.15) leads to

$$
\begin{aligned}
x(t) & =x\left(t_{0}\right)+\int_{t_{0}}^{t} x^{\prime}(s) d s \\
& \leq x\left(t_{0}\right)+\sup _{t \in R}\left\{-\frac{a(t) M}{b(t)+M}+\frac{1}{e} \sum_{j=1}^{m} \frac{\beta_{j}(t)}{\gamma_{j}(t)}\right\}\left(t-t_{0}\right), \forall t \geq t_{0},
\end{aligned}
$$

and

$$
\lim _{t \rightarrow+\infty} x(t)=-\infty
$$

which contradicts with (3.11). Hence, (3.13) holds. We claim:

$$
x(t)<M \text { for all } t \in\left[t_{4}, \eta(\varphi)\right) \text {, and } \eta(\varphi)=+\infty .
$$

Suppose, for the sake of contradiction, there exists $t_{5} \in\left(t_{4}, \eta(\varphi)\right)$ such that

$$
x\left(t_{5}\right)=M, \quad x(t)<M \text { for all } t \in\left[t_{4}, t_{5}\right) .
$$

Calculating the derivative of $x(t)$, together with the fact that $\sup _{x \in R} x e^{-x}=\frac{1}{e}$, (1.2), (2.2) and (3.17) imply that

$$
\begin{aligned}
0 & \leq x^{\prime}\left(t_{5}\right) \\
& =-\frac{a\left(t_{5}\right) M}{b\left(t_{5}\right)+M}+\sum_{j=1}^{m} \frac{\beta_{j}\left(t_{5}\right)}{\gamma_{j}\left(t_{5}\right)} \gamma_{j}\left(t_{5}\right) x\left(t_{5}-\tau_{j}\left(t_{5}\right)\right) e^{-\gamma_{j}\left(t_{5}\right) x\left(t_{5}-\tau_{j}\left(t_{5}\right)\right)} \\
& \leq-\frac{a\left(t_{5}\right) M}{b\left(t_{5}\right)+M}+\frac{1}{e} \sum_{j=1}^{m} \frac{\beta_{j}\left(t_{5}\right)}{\gamma_{j}\left(t_{5}\right)} \\
& <0
\end{aligned}
$$

which is a contradiction and implies that (3.16) holds.

Furthermore, we prove that there exists a positive constant $l$ such that

$$
\liminf _{t \rightarrow+\infty} x(t)=l .
$$

Otherwise, we assume that $\liminf _{t \rightarrow+\infty} x(t)=0$. For each $t \geq t_{0}$, we define

$$
m(t)=\max \left\{\xi: \xi \leq t, x(\xi)=\min _{t_{0} \leq s \leq t} x(s)\right\} .
$$

Observe that $m(t) \rightarrow+\infty$ as $t \rightarrow+\infty$ and that

$$
\lim _{t \rightarrow+\infty} x(m(t))=0 .
$$


However, $x(m(t))=\min _{t_{0} \leq s \leq t} x(s)$, and so $x^{\prime}(m(t)) \leq 0$ for all $m(t)>t_{0}$. According to (1.2), we have

$$
\begin{aligned}
0 \geq & x^{\prime}(m(t)) \\
= & -\frac{a(m(t)) x(m(t))}{b(m(t))+x(m(t))} \\
& +\sum_{j=1}^{m} \beta_{j}(m(t)) x\left(m(t)-\tau_{j}(m(t))\right) e^{-\gamma_{j}(m(t)) x\left(m(t)-\tau_{j}(m(t))\right)} \\
\geq & -\frac{a(m(t)) x(m(t))}{b(m(t))} \\
& +\sum_{j=1}^{m} \beta_{j}(m(t)) x\left(m(t)-\tau_{j}(m(t))\right) e^{-\gamma_{j}(m(t)) x\left(m(t)-\tau_{j}(m(t))\right)},
\end{aligned}
$$

and consequently,

$$
\begin{aligned}
\frac{a(m(t)) x(m(t))}{b(m(t))} & \geq \sum_{j=1}^{m} \beta_{j}(m(t)) x\left(m(t)-\tau_{j}(m(t))\right) e^{-\gamma_{j}(m(t)) x\left(m(t)-\tau_{j}(m(t))\right)} \\
& \geq \beta_{j}(m(t)) x\left(m(t)-\tau_{j}(m(t))\right) e^{-\gamma_{j}(m(t)) x\left(m(t)-\tau_{j}(m(t))\right)},
\end{aligned}
$$

where $m(t)>t_{0}, j=1,2, \ldots, m$. This, together with (3.19), implies that

$$
\lim _{t \rightarrow+\infty} x\left(m(t)-\tau_{j}(m(t))\right)=0, j=1,2, \ldots, m .
$$

Noting that the continuities and boundedness of the functions $a(t), b(t)$ and $\beta_{j}(t)$, we can select a sequence $\left\{t_{n}\right\}_{n=1}^{+\infty}$ such that

$$
\begin{aligned}
& \lim _{n \rightarrow+\infty} t_{n}=+\infty, \lim _{n \rightarrow+\infty} x\left(m\left(t_{n}\right)\right)=0, \\
& \lim _{n \rightarrow+\infty} \frac{\beta_{j}\left(m\left(t_{n}\right)\right) b\left(m\left(t_{n}\right)\right)}{a\left(m\left(t_{n}\right)\right)}=a_{j}^{*}, j=1,2, \ldots, m .
\end{aligned}
$$

In view of (3.20), we get

$$
\begin{aligned}
& \frac{a\left(m\left(t_{n}\right)\right)}{b\left(m\left(t_{n}\right)\right)} \\
\geq & \sum_{j=1}^{m} \beta_{j}\left(m\left(t_{n}\right)\right) \frac{x\left(m\left(t_{n}\right)-\tau_{j}\left(m\left(t_{n}\right)\right)\right) e^{-\gamma_{j}\left(m\left(t_{n}\right)\right) x\left(m\left(t_{n}\right)-\tau_{j}\left(m\left(t_{n}\right)\right)\right)}}{x\left(m\left(t_{n}\right)\right)} \\
\geq & \sum_{j=1}^{m} \beta_{j}\left(m\left(t_{n}\right)\right) \frac{x\left(m\left(t_{n}\right)-\tau_{j}\left(m\left(t_{n}\right)\right)\right) e^{-\gamma_{j}^{+} x\left(m\left(t_{n}\right)-\tau_{j}\left(m\left(t_{n}\right)\right)\right)}}{x\left(m\left(t_{n}\right)-\tau_{j}\left(m\left(t_{n}\right)\right)\right)} \\
= & \sum_{j=1}^{m} \beta_{j}\left(m\left(t_{n}\right)\right) e^{-\gamma_{j}^{+} x\left(m\left(t_{n}\right)-\tau_{j}\left(m\left(t_{n}\right)\right)\right)}
\end{aligned}
$$


and

$$
1 \geq \sum_{j=1}^{m} \frac{\beta_{j}\left(m\left(t_{n}\right)\right) b\left(m\left(t_{n}\right)\right)}{a\left(m\left(t_{n}\right)\right)} e^{-\gamma_{j}^{+} x\left(m\left(t_{n}\right)-\tau_{j}\left(m\left(t_{n}\right)\right)\right)} .
$$

Letting $n \rightarrow+\infty,(3.21)$, (3.22) and (3.23) imply that

$$
\begin{aligned}
1 & \geq \sum_{j=1}^{m} \lim _{n \rightarrow+\infty} \frac{\beta_{j}\left(m\left(t_{n}\right)\right) b\left(m\left(t_{n}\right)\right)}{a\left(m\left(t_{n}\right)\right)} \lim _{n \rightarrow+\infty} e^{-\gamma_{j}^{+} x\left(m\left(t_{n}\right)-\tau_{j}\left(m\left(t_{n}\right)\right)\right)} \\
& =\lim _{n \rightarrow+\infty} \sum_{j=1}^{m} \frac{\beta_{j}\left(m\left(t_{n}\right)\right) b\left(m\left(t_{n}\right)\right)}{a\left(m\left(t_{n}\right)\right)} \\
& \geq \liminf _{t \rightarrow+\infty} \sum_{j=1}^{m} \frac{\beta_{j}(t) b(t)}{a(t)} .
\end{aligned}
$$

From (2.2), we get

$$
\begin{aligned}
0 & <\inf _{t \in R, s \in[0, \kappa]}\left\{-\frac{a(t)}{b(t)+s}+\sum_{j=1}^{m} \frac{\beta_{j}(t)}{\gamma_{j}(t)} e^{-s}\right\} \\
& \leq \inf _{t \in R}\left\{-\frac{a(t)}{b(t)}+\sum_{j=1}^{m} \frac{\beta_{j}(t)}{\gamma_{j}(t)}\right\} \\
& \leq \inf _{t \in R}\left\{-\frac{a(t)}{b(t)}+\sum_{j=1}^{m} \beta_{j}(t)\right\},
\end{aligned}
$$

and

$$
0<\inf _{t \in R}\left\{\frac{a(t)}{b(t)}\left[-1+\sum_{j=1}^{m} \frac{\beta_{j}(t) b(t)}{a(t)}\right]\right\} \quad \text { for all } t \in R,
$$

which contradicts to (3.24). Hence, (3.18) holds.

To prove (3.12), it is sufficient to show $l>\kappa$. If not, we assume that $l \leq \kappa$.

By the fluctuation lemma [26, Lemma A.1], there exists a sequence a sequence $\left\{t_{k}\right\}_{k=1}^{+\infty}$ such that

$t_{k} \rightarrow+\infty, x\left(t_{k} ; t_{0}, \varphi\right) \rightarrow l$, and $x^{\prime}\left(t_{k} ; t_{0}, \varphi\right)=f\left(t_{k}, x_{t_{k}}\left(t_{0}, \varphi\right)\right) \rightarrow 0$, as $k \rightarrow+\infty$.

Since $\left\{x_{t_{k}}\left(t_{0}, \varphi\right)\right\}_{k=1}^{+\infty}$ is bounded and equicontinuous, by Ascoli-Arzelà Theorem, for a subsequence, still denoted by $\left\{x_{t_{k}}\left(t_{0}, \varphi\right)\right\}_{k=1}^{+\infty}$, we have

$$
x_{t_{k}}\left(t_{0}, \varphi\right) \longrightarrow \varphi^{*} \text { for some } \varphi^{*} \in C([-r, 0],(0,+\infty)) .
$$

From (3.16), we get

$$
\varphi^{*}(0)=l \leq \varphi^{*}(s) \leq M \text { for } s \in[-r, 0] .
$$

By the boundedness of $\left\{\tau_{j}\left(t_{k}\right)\right\}_{k=1}^{+\infty}$, there is a subsequence of $\left\{t_{k}\right\}_{k=1}^{+\infty}$, still denoted by $\left\{t_{k}\right\}_{k=1}^{+\infty}$, which converges to a point $\tau_{j}^{*} \in\left[\tau_{j}^{-}, \tau_{j}^{+}\right]$with $j=$ 
$1,2, \ldots, m$. Similarly, we can also suppose that

$$
\left\{\begin{array}{l}
\lim _{k \rightarrow+\infty} a\left(t_{k}\right)=a^{*} \in\left[a^{-}, a^{+}\right], \lim _{k \rightarrow+\infty} b\left(t_{k}\right)=b^{*} \in\left[b^{-}, b^{+}\right], \\
\lim _{k \rightarrow+\infty} \beta_{j}=\beta_{j}^{*} \in\left[\beta_{j}^{-}, \beta_{j}^{+}\right], \lim _{k \rightarrow+\infty} \gamma_{j}=\gamma_{j}^{*} \in\left[\gamma_{j}^{-}, \gamma_{j}^{+}\right], j=1,2, \ldots, m .
\end{array}\right.
$$

Hence,

$$
f\left(t_{k}, x_{t_{k}}\left(t_{0}, \varphi\right)\right) \rightarrow \Lambda \text { as } k \rightarrow+\infty
$$

with

$$
\Lambda=-\frac{a^{*} \varphi^{*}(0)}{b^{*}+\varphi^{*}(0)}+\sum_{j=1}^{m} \beta_{j}^{*} \varphi^{*}\left(-\tau_{j}^{*}\right) e^{-\gamma_{j}^{*} \varphi^{*}\left(-\tau_{j}^{*}\right)} .
$$

According to (1.8), (1.9), (2.1), (2.2) and the fact that

$$
0<l \leq \kappa, \quad l \leq \gamma_{j}^{*} \varphi^{*}\left(-\tau_{j}^{*}\right) \leq \gamma_{j}^{+} M \leq \widetilde{\kappa}, j=1,2, \ldots, m,
$$

we obtain

$$
\begin{aligned}
\Lambda & =-\frac{a^{*} l}{b^{*}+l}+\sum_{j=1}^{m} \frac{\beta_{j}^{*}}{\gamma_{j}^{*}} \gamma_{j}^{*} \varphi^{*}\left(-\tau_{j}^{*}\right) e^{-\gamma_{j}^{*} \varphi^{*}\left(-\tau_{j}^{*}\right)} \\
& \geq-\frac{a^{*} l}{b^{*}+l}+\sum_{j=1}^{m} \frac{\beta_{j}^{*}}{\gamma_{j}^{*}} l e^{-l} \\
& =l\left[-\frac{a^{*}}{b^{*}+l}+\sum_{j=1}^{m} \frac{\beta_{j}^{*}}{\gamma_{j}^{*}} e^{-l}\right] \\
& \geq l \min _{t \in[0, T], s \in[0, \kappa]}\left\{-\frac{a(t)}{b(t)+s}+\sum_{j=1}^{m} \frac{\beta_{j}(t)}{\gamma_{j}(t)} e^{-s}\right\} \\
& >0, \quad{ }^{-s}
\end{aligned}
$$

which contradicts $(3.25)$ and implies $l>\kappa$.

Finally, we prove that $x^{*}(t)$ is globally exponentially stable.

Set $y(t)=x(t)-x^{*}(t)$, where $t \in\left[t_{0}-r,+\infty\right)$. Then

$$
\begin{aligned}
& y^{\prime}(t) \\
= & -\left[\frac{a(t) x(t)}{b(t)+x(t)}-\frac{a(t) x^{*}(t)}{b(t)+x^{*}(t)}\right] \\
& +\sum_{j=1}^{m} \beta_{j}(t)\left[x\left(t-\tau_{j}(t)\right) e^{-\gamma_{j}(t) x\left(t-\tau_{j}(t)\right)}-x^{*}\left(t-\tau_{j}(t)\right) e^{-\gamma_{j}(t) x^{*}\left(t-\tau_{j}(t)\right)}\right] .
\end{aligned}
$$

We consider the Lyapunov functional

$$
V(t)=|y(t)| e^{\lambda t}, \text { where } \lambda \text { is defined in (2.9). }
$$


Calculating the upper left derivative of $V(t)$ along the solution $y(t)$ of $(3.31)$, we have

$$
\begin{aligned}
D^{-}(V(t)) \leq & -\left[\frac{a(t) x(t)}{b(t)+x(t)}-\frac{a(t) x^{*}(t)}{b(t)+x^{*}(t)}\right] \operatorname{sgn}\left(x(t)-x^{*}(t)\right) e^{\lambda t} \\
& +\sum_{j=1}^{m} \beta_{j}(t) \mid x\left(t-\tau_{j}(t)\right) e^{-\gamma_{j}(t) x\left(t-\tau_{j}(t)\right)} \\
& -x^{*}\left(t-\tau_{j}(t)\right) e^{-\gamma_{j}(t) x^{*}\left(t-\tau_{j}(t)\right)}\left|e^{\lambda t}+\lambda\right| y(t) \mid e^{\lambda t} \text { for all } t>t_{0} .
\end{aligned}
$$

We claim that

$$
\begin{aligned}
V(t) & =|y(t)| e^{\lambda t} \\
& =\left|x(t)-x^{*}(t)\right| e^{\lambda t} \\
& <e^{\lambda\left(t_{\varphi}+r\right)}\left(\max _{t \in\left[t_{0}-r, t_{\varphi}+r\right]}\left|x(t)-x^{*}(t)\right|+1\right) \\
& :=K \text { for all } t>t_{\varphi}+r,
\end{aligned}
$$

Contrarily, there must exist $t_{*}>t_{\varphi}+r$ such that

$$
V\left(t_{*}\right)=K \text { and } V(t)<K \text { for all } t \in\left[t_{0}-r, t_{*}\right) .
$$

Since $x(t) \geq \kappa$ and $x^{*}(t) \geq \kappa$ for all $t \geq t_{\varphi}$. Together with (2.14), (2.15), (3.31) and (3.33), we obtain

$$
\begin{aligned}
0 \leq & D^{-}\left(V\left(t_{*}\right)\right) \\
\leq & -\left[\frac{a\left(t_{*}\right) x\left(t_{*}\right)}{b\left(t_{*}\right)+x\left(t_{*}\right)}-\frac{a\left(t_{*}\right) x^{*}\left(t_{*}\right)}{b\left(t_{*}\right)+x^{*}\left(t_{*}\right)}\right] \operatorname{sgn}\left(x\left(t_{*}\right)-x^{*}\left(t_{*}\right)\right) e^{\lambda t_{*}} \\
& +\sum_{j=1}^{m} \beta_{j}\left(t_{*}\right) \mid x\left(t_{*}-\tau_{j}\left(t_{*}\right)\right) e^{-\gamma_{j}\left(t_{*}\right) x\left(t_{*}-\tau_{j}\left(t_{*}\right)\right)} \\
& -x^{*}\left(t_{*}-\tau_{j}\left(t_{*}\right)\right) e^{-\gamma_{j}\left(t_{*}\right) x^{*}\left(t_{*}-\tau_{j}\left(t_{*}\right)\right)}\left|e^{\lambda t_{*}}+\lambda\right| y\left(t_{*}\right) \mid e^{\lambda t_{*}} \\
\leq & -\frac{a\left(t_{*}\right) b\left(t_{*}\right)}{\left(b\left(t_{*}\right)+M\right)^{2}}\left|y\left(t_{*}\right)\right| e^{\lambda t_{*}}+\sum_{j=1}^{m} \beta_{j}\left(t_{*}\right) \mid x\left(t_{*}-\tau_{j}\left(t_{*}\right)\right) e^{-\gamma_{j}\left(t_{*}\right) x\left(t_{*}-\tau_{j}\left(t_{*}\right)\right)} \\
& -x^{*}\left(t_{*}-\tau_{j}\left(t_{*}\right)\right) e^{-\gamma_{j}\left(t_{*}\right) x^{*}\left(t_{*}-\tau_{j}\left(t_{*}\right)\right)}\left|e^{\lambda t_{*}}+\lambda\right| y\left(t_{*}\right) \mid e^{\lambda t_{*}} \\
= & -\left[\frac{a\left(t_{*}\right) b\left(t_{*}\right)}{\left(b\left(t_{*}\right)+M\right)^{2}}-\lambda\right]\left|y\left(t_{*}\right)\right| e^{\lambda t_{*}} \\
& +\sum_{j=1}^{m} \frac{\beta_{j}\left(t_{*}\right)}{\gamma_{j}\left(t_{*}\right)} \mid \gamma_{j}\left(t_{*}\right) x\left(t_{*}-\tau_{j}\left(t_{*}\right)\right) e^{-\gamma_{j}\left(t_{*}\right) x\left(t_{*}-\tau_{j}\left(t_{*}\right)\right)} \\
& -\gamma_{j}\left(t_{*}\right) x^{*}\left(t_{*}-\tau_{j}\left(t_{*}\right)\right) e^{-\gamma_{j}\left(t_{*}\right) x^{*}\left(t_{*}-\tau_{j}\left(t_{*}\right)\right)} \mid e^{\lambda t_{*}} \\
\leq & -\left[\frac{a\left(t_{*}\right) b\left(t_{*}\right)}{\left(b\left(t_{*}\right)+M\right)^{2}}-\lambda\right]\left|y\left(t_{*}\right)\right| e^{\lambda t_{*}}
\end{aligned}
$$




$$
\begin{aligned}
& +\sum_{j=1}^{m} \beta_{j}\left(t_{*}\right) \frac{1}{e^{2}}\left|y\left(t_{*}-\tau_{j}\left(t_{*}\right)\right)\right| e^{\lambda\left(t_{*}-\tau_{j}\left(t_{*}\right)\right)} e^{\lambda \tau_{j}\left(t_{*}\right)} \\
\leq & \left\{-\left[\frac{a\left(t_{*}\right) b\left(t_{*}\right)}{\left(b\left(t_{*}\right)+M\right)^{2}}-\lambda\right]+\sum_{j=1}^{m} \beta_{j}\left(t_{*}\right) \frac{1}{e^{2}} e^{\lambda r}\right\} K .
\end{aligned}
$$

Thus,

$$
0 \leq-\left[\frac{a\left(t_{*}\right) b\left(t_{*}\right)}{\left(b\left(t_{*}\right)+M\right)^{2}}-\lambda\right]+\sum_{j=1}^{m} \beta_{j}\left(t_{*}\right) \frac{1}{e^{2}} e^{\lambda r},
$$

which contradicts with (2.9). Hence, (3.32) holds. It follows that

$$
|y(t)|<K e^{-\lambda t} \text { for all } t>t_{\varphi}+r .
$$

This completes the proof.

\section{An example}

In this section, we present an example to check the validity of our results we obtained in the previous sections.

Example 4.1. Consider the following Nicholson's blowflies model with a nonlinear density-dependent mortality term:

(4.1) $x^{\prime}(t)=-\frac{0.6951934 x(t)}{0.7537127+x(t)}+\frac{100+\sin \sqrt{5} t}{100+\cos \sqrt{3} t} x\left(t-2 e^{\sin ^{4} t}\right) e^{-x\left(t-2 e^{\sin ^{4} t}\right)}$.

Obviously, $r=2 e, a^{-}=a^{+}=0.6951934, b^{-}=b^{+}=0.7537127, \beta_{1}^{-} \geq \frac{99}{101}$, $\beta_{j}^{+} \leq \frac{101}{99}, \gamma_{1}^{-}=\gamma_{1}^{+}=1$. From (1.7), (1.8), $\tilde{\kappa}>1$ and $\kappa e^{-\kappa}=\widetilde{\kappa} e^{-\widetilde{\kappa}}$, we obtain $\kappa \approx 0.7215355, \tilde{\kappa} \approx 1.342276$.

Let $M=1.087308$, we get

$$
\begin{aligned}
\frac{a^{-} M}{b^{+}+M} & =\frac{0.6951934 \times 1.087308}{0.7537127+1.087308} \approx 0.4105817 \\
\frac{\beta_{1}^{+}}{\gamma_{1}^{-}} \frac{1}{e} \leq \frac{101}{99} \frac{1}{e} \approx 0.3753113 & \\
\geq & \min _{t \in[0, T], s \in[0, \kappa]}\left\{-\frac{a(t)}{b(t)+s}+\frac{\beta_{1}(t)}{\gamma_{1}(t)} e^{-s}\right\} \\
& =\min _{t \in[0, T], s \in[0, \kappa]}\left\{-\frac{0.6951934}{0.7537127+s}+\frac{99}{101} e^{-s\}}\right. \\
\frac{\min ^{-} b^{-}}{\left(b^{+}+[0, \kappa]\right.}\left\{-\frac{0.6951934}{0.7537127+s}+\frac{99}{101} e^{-s}\right\} \approx 0.005143492 & =\frac{6951934 \times 0.7537127}{(0.7537127+1.087308)^{2}} \approx 0.1545945 \\
\beta_{1}^{+} \frac{1}{e^{2}} \leq & \frac{101}{99} \frac{1}{e^{2}} \approx 0.1380693
\end{aligned}
$$


which implies that the Nicholson's blowflies model (4.1) satisfies (2.1), (2.2) and (2.7). Hence, from Theorems 3.2, equation (4.1) has exactly one positive almost periodic solution $x^{*}(t)$. Moreover, $x^{*}(t)$ is globally exponentially stable. This fact is verified by the numerical simulation in Figs. 1-3.

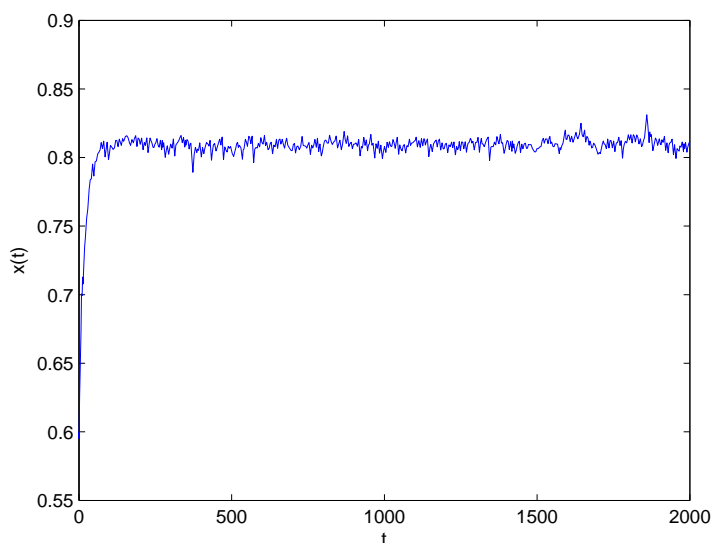

FIG. 1. Numerical solution $x(t)$ of equation (4.1) for initial value $\varphi(s) \equiv$ $0.6, s \in[-2 e, 0]$.

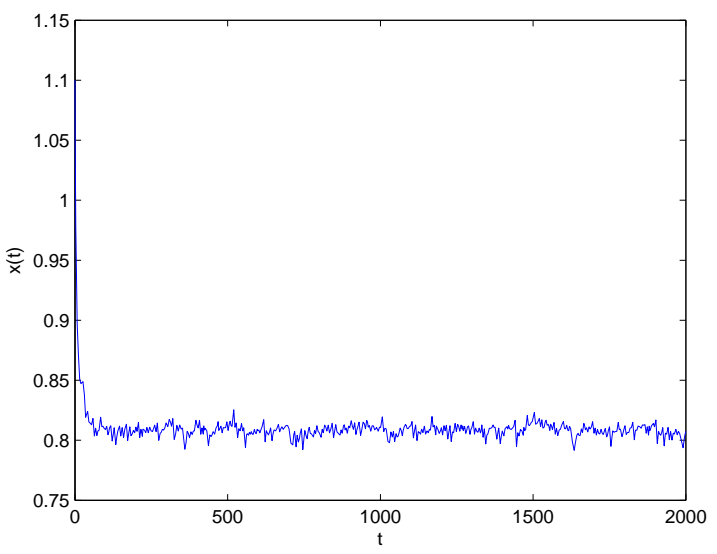

FIG. 2. Numerical solution $x(t)$ of equation (4.1) for initial value $\varphi(s) \equiv$ $1.1, s \in[-2 e, 0]$. 


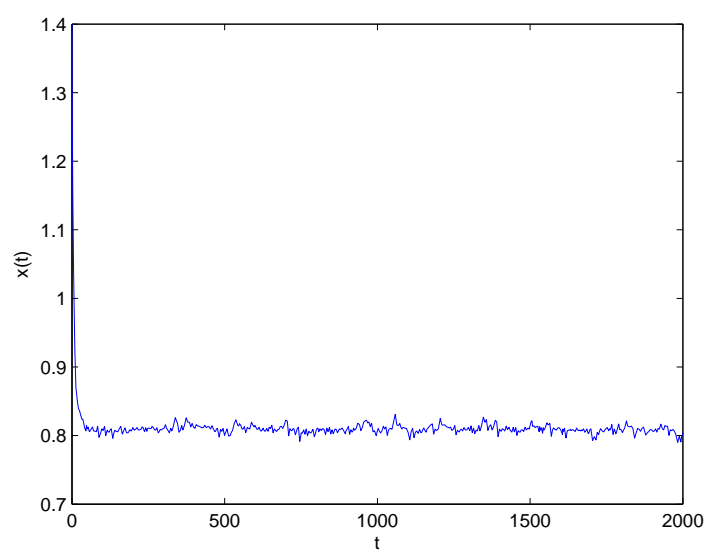

FIG. 3. Numerical solution $x(t)$ of equation (4.1) for initial value $\varphi(s) \equiv$ $1.4, s \in[-2 e, 0]$.

Remark 4.1. Recently, H. Ding et al. [6] and W. Wang [28] gave the local existence of positive almost periodic solutions of Nicholson's blowflies model with a nonlinear density-dependent mortality term $a(t)-b(t) e^{-x(t)}$ and a harvesting term, respectively. Unfortunately, the global exponential convergence for the positive almost periodic solutions of Nicholson's blowflies model have not been studied in [6, 28]. Most recently, B. Liu [16] employed a novel proof to establish some criteria to guarantee the existence and exponential stability of positive periodic solutions for Nicholson's blowflies model with time-varying coefficients and delays. And the author also indicated that it is difficult to establish criteria ensuring global exponential stability of positive almost periodic solutions for Nicholson's blowflies model. For all we know, there is no research on the problems of positive almost periodic solutions of Nicholson's blowflies model with a nonlinear density-dependent mortality term $\frac{a(t) x(t)}{b(t)+x(t)}$. Thus, all the results in the references $[2,4,5,6,11,12,13,14,15,17,18,21,24,28,32]$ and $[3,19,27,30]$ cannot be applicable to prove that all the solutions of $(4.1)$ with initial value $\varphi \in C_{+}$and $\varphi(0)>0$ converge exponentially to the positive almost periodic solution. Moreover, in this present paper, we give a novel proof to establish some criteria to guarantee the global exponential stability of almost periodic solutions for Nicholson's blowflies model with a nonlinear density-dependent mortality term.

Acknowledgement. The author would like to express the sincere appreciation to the editor and reviewer for their helpful comments in improving the presentation and quality of the paper. In particular, the authors expresses the sincere gratitude to Prof. Jianli Li for the helpful discussion when this work is carried out. 


\section{References}

[1] L. Berezansky, E. Braverman, and L. Idels, Nicholson's blowflies differential equations revisited: Main results and open problems, Appl. Math. Model. 34 (2010), no. 6, 14051417.

[2] W. Chen, Permanence for Nicholson-type delay systems with patch structure and nonlinear density-dependent mortality terms, Electron. J. Qual. Theory Differ. Equ. 2012 (2012), no. 73, 1-14.

[3] W. Chen and B. Liu, Positive almost periodic solution for a class of Nicholson's blowflies model with multiple time-varying delays, J. Comput. Appl. Math. 235 (2011), no. 8, 2090-2097.

[4] W. Chen and L. Wang, Positive periodic solutions of Nicholson-type delay systems with nonlinear density-dependent mortality terms, Abstr. Appl. Anal. 2012 (2012), Art. ID 843178, 13 pp.

[5] Y. Chen, Periodic solutions of delayed periodic Nicholson's blowflies models, Can. Appl. Math. Q. 11 (2003), no. 1, 23-28.

[6] H. Ding and J. J. Nieto, A new approach for positive almost periodic solutions to a class of Nicholson's blowflies model, J. Comput. Appl. Math. 253 (2013), 249-254.

[7] A. M. Fink, Almost Periodic Differential Equations, in: Lecture Notes in Mathematics, vol. 377, Springer, Berlin, 1974.

[8] W. Gurney, S. Blythe, and R. Nisbet, Nicholsons blowflies revisited, Nature 287 (1980), $17-21$

[9] J. K. Hale and S. M. Verduyn Lunel, Introduction to Functional Differential Equations, Springer-Verlag, New York, 1993.

[10] C. Y. He, Almost Periodic Differential Equation, Higher Education Publishing House, Beijing, 1992 (in Chinese).

[11] X. Hou, L. Duan, and Z. Huang, Permanence and periodic solutions for a class of delay Nicholson's blowflies models, Appl. Math. Model. 37 (2013), no. 3, 1537-1544.

[12] J. Li and C. Du, Existence of positive periodic solutions for a generalized Nicholson's blowflies model, J. Comput. Appl. Math. 221 (2008), no. 1, 226-233.

[13] B. Liu, The existence and uniqueness of positive periodic solutions of Nicholson-type delay systems, Nonlinear Anal. Real World Appl. 12 (2011), no. 6, 3145-3151.

[14] _ Permanence for a delayed Nicholson's blowflies model with a nonlinear densitydependent mortality term, Ann. Polon. Math. 101 (2011), no. 2, 123-128.

[15] _ Positive periodic solutions for a nonlinear density-dependent mortality Nicholson's blowflies model, Kodai Math. J. 37 (2014), no. 1, 157-173.

[16] - Global exponential stability of positive periodic solutions for a delayed Nicholson's blowflies model, J. Math. Anal. Appl. 412 (2014), no. 1, 212-221.

[17] B. Liu and S. Gong, Permanence for Nicholson-type delay systems with nonlinear density-dependent mortality terms, Nonlinear Anal. Real World Appl. 12 (2011), no. 4, 1931-1937.

[18] X. Liu and J. Meng, The positive almost periodic solution for Nicholson-type delay systems with linear harvesting terms, Appl. Math. Model. 36 (2012), no. 7, 3289-3298.

[19] F. Long, Positive almost periodic solution for a class of Nicholson's blowflies model with a linear harvesting term, Nonlinear Anal. Real World Appl. 13 (2012), no. 2, 686-693.

[20] F. Long and B. Liu, Existence and uniqueness of positive periodic solutions of delayed Nicholson's blowflies models, Ann. Polon. Math. 103 (2012), no. 3, 217-228.

[21] F. Long and M. Yang, Positive periodic solutions of delayed Nicholson's blowflies model with a linear harvesting term, Electron. J. Qual. Theory Differ. Equ. 2011 (2011), no. $41,1-11$.

[22] A. Nicholson, An outline of the dynamics of animal populations, Aust. J. Zool. 2 (1954), 9-65. 
[23] R. Nisbet and W. Gurney, Modelling Fluctuating Populations, John Wiley and Sons, NY, 1982.

[24] S. Saker and S. Agarwal, Oscillation and global attractivity in a periodic Nicholson's blowflies model, Math. Comput. Modelling 35 (2002), no. 7-8, 719-731.

[25] H. L. Smith, Monotone Dynamical Systems, Math. Surveys Monogr., Amer. Math. Soc., Providence, RI, 1995.

[26] - An Introduction to Delay Differential Equations with Applications to the Life Sciences, Springer New York, 2011.

[27] L. Wang, Almost periodic solution for Nicholsons blowflies model with patch structure and linear harvesting terms, Appl. Math. Model. 37 (2013), 2153-2165.

[28] W. Wang, Positive periodic solutions of delayed Nicholson's blowflies models with a nonlinear density-dependent mortality term, Appl. Math. Model. 36 (2012), no. 10, 4708-4713.

[29] _ Exponential extinction of Nicholson's blowflies system with nonlinear densitydependent mortality terms, Abstr. Appl. Anal. 2012 (2012), Art. ID 302065, 15 pp; doi:10.1155/2012/302065.

[30] W. Wang, L. Wang, and W. Chen, Existence and exponential stability of positive almost periodic solution for Nicholson-type delay systems, Nonlinear Anal. Real World Appl. 12 (2011), no. 4, 1938-1949.

[31] Z. Zheng, H. Ding, and Gaston M. $N^{\prime}$ Guérékata, The space of continuous periodic functions is a set of first category in $A P(X)$, J. Funct. Spac. and Appl. 2013(275702) (2013), 1-3.

[32] Q. Zhou, The positive periodic solution for Nicholson-type delay system with linear harvesting terms, Appl. Math. Model. 37 (2013), no. 8, 5581-5590.

Department of Mathematics

Xiangnan College

Chenzhou, Hunan 423000, P. R. China

E-mail address: xuyanliling@aliyun.com 